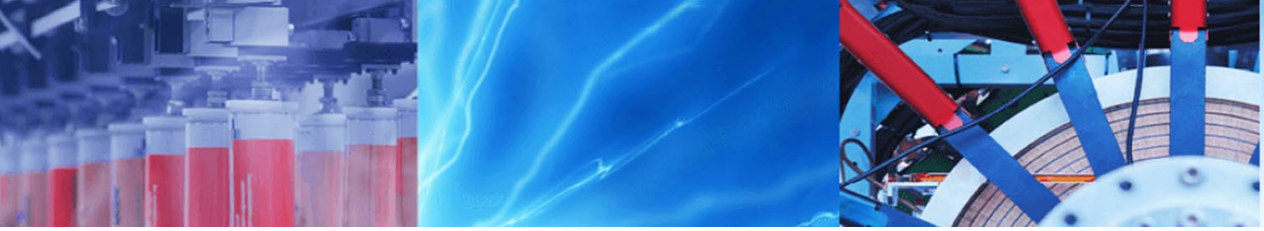

Research Article

\title{
The impact of segmentation method on the aggregate goodness-of-fit measurements of non-linear crash prediction models
}

\author{
Mahdi Akbari ${ }^{1}$. Gholamali Shafabakhsh ${ }^{1} \cdot$ Mohammad Reza Ahadi ${ }^{2}$
}

Received: 21 February 2020 / Accepted: 26 August 2020 / Published online: 9 September 2020

(c) Springer Nature Switzerland AG 2020

\begin{abstract}
This study investigates the influence of segmentation type on the goodness-of-fit and the significance of crash prediction accuracy of non-linear crash prediction models. The models are developed regarding run-off-road accidents, pavement distresses, and roughness. The nonlinear negative binomial regression models are employed to establish a relationship between run-off-road accident frequency and other independent variables. Results show that homogeneous segmentation have fewer errors and is preferred against fixed-length segmentation, whereas the model development of fixed-length segments defines enhanced goodness-of-fit values. The comparison of the suggested nonlinear models confirm that it is better to execute the segmentation process for studying the crash prediction models of some specific explanatory variables, like pavement characteristics variables.
\end{abstract}

Keywords Highway segmentation · Pavement physical characteristics · Crash prediction models · Run-off-road accidents $\cdot$ Nonlinear negative binomial regression

\section{Introduction}

Crash prediction models (CPMs) as a mathematical equations are useful measures that developed to improve road safety by analyzing and identifying hot spots. CPMs link road safety performance and crash factors by considering a function of variety explanatory variables, including the roadway, the roadway environment, vehicles and road users' behavior. These models are known as safety performance functions (SPFs). Significant researches should be implemented on the development of CPMs for the estimation of the predicted accident frequencies or crash rates on road networks.

In developing CPMs for roadway segments, there exist two modes in terms of segmentation: either homogeneous segments or fixed-length segments. In the case of homogeneous segments, the length of segments should be determined based on the change in some explanatory variables. In other words, it is assumed that each segment possesses some uniform properties regarding several features and these changes are constant along the length of each segment such as homogeneous segments in terms of geometric design (located in curves or tangents) or segments with specified roughness domain. However, in the case of fixed-length segments, it is assumed that the length of all segments is fixed and not subject to changes in explanatory variables such as $100-\mathrm{m}$ segments, $1-\mathrm{km}$ segments, etc.

Several studies have been conducted on the two route segmentation approaches. Some researchers used the fixed-length segments for the development of CPMs [1-7], and on the other hand, some researchers also considered homogeneous segments in this regard [8-14].

Shankar et al. [1] pointed out that the defects in the implementation of fixed-length segmentation were much smaller than when homogeneous segments were used.

Gholamali Shafabakhsh, ghshafabakhsh@semnan.ac.ir| ${ }^{1}$ Faculty of Civil Engineering, Semnan University, Semnan, Iran. ${ }^{2}$ Transportation Research Institute, Ministry of Roads and Urban Development, Tehran, Iran. 
Therefore, the following question arises: should segmentation, following the viewpoint of Shankar et al. [1], include fixed-length segments or according to recent researches, be implemented based on homogeneous segments?

Ambros et al. [15] investigated the main aspects of developing crash prediction models (CPMs) and discussed the road network segmentation concepts. They explained that although some researches set fixed lengths [5-7] and the others set homogeneous segments [11-13], division of road network into segments, in practice, is likely to be dictated by structure of road infrastructure databases. They introduced the generalized linear model (GLM) to develop CPMs. Akbari et al. [14] developed tree types of CPMs include GLMs (the Poisson regression (PR) and negative binomial regression (NBR)), and non-linear negative binomial regression models to consider the impact of pavement physical characteristics on the frequency of single-vehicle run-off-road (ROR) crashes. Although Ambros et al. (2018) introduced the GLMs as a traditional functional form for CPMs [15], the results of Akbari et al. [14] study declared that the non-linear proposed CPM gives better fitness than GLMs.

The purpose of this paper is to investigate the effect of different segmentation methods on the aggregate goodness-of-fit measurements (external errors) of CPMs for separated two-lane roadways by considering the MAE and RMSE measures. The proposed nonlinear negative binomial regression (NBR) models of this paper are developed for fixed-length and homogeneous segments. In fact, this study formulated and compared practicable CPMs (based on homogeneous and fixed-length segmentation), which could describe the relationship between the expected run-off-road (ROR) accident frequency and the explanatory variables. In addition, safety effects of explanatory and independent variables on their domains are examined and presented in the current study. The models that have been proposed so far are mainly for investigating relationship between the expected ROR accident frequency and the explanatory variables. Although GLMs are common in CPM development studies, some studies needed nonlinear functional forms to develop CPMs. So this paper is focused more on developing nonlinear negative binomial regression models which their residuals are affected by the segmentation methods and simultaneously the other independent variables' data are the same for all models. The main independent variables that introduced to the CPMs are accident frequency data, exposure parameters (include AADT and length of segment), pavement condition variable (includes $\mathrm{PCl}$ ) and riding quality variables (include IRI and RN).

In Sect. 2, the literature review of the relevant studies are reviewed. Section 3 presents the data description and in Sect. 4, the methodological issues and processes associated with the establishment, fitting, and evaluation of nonlinear NBR CPMs are explained. Section 5 explains the results of modeling and in Sect. 6, the effects of different segmentation methods on aggregate goodness of fit measurements (external errors) of models and safety effects of independent variables are discussed. Finally, Sect. 7 presents the conclusion of the study.

\section{Literature review}

Shankar et al. [1] concluded in their research that homogeneous segments of various lengths might exacerbate problems of heteroscedasticity, or even reduce the efficiency of the model estimation, which is mainly of greater importance in the case of the negative binomial distribution, as the gamma-distributed error term is incorporated in it.

However, there exists a significant number of studies in which homogeneous segments were used. In these studies, the exposure parameter included in CPMs for roadway segments consisted of combinations of two variables: Annual Average Daily Traffic (AADT) and segment length $[16,17]$. However, it should be noted that the exposure variables in some cases have been included as offset and in some other cases as a predictor, but none of them is often assumed as fixed.

Investigating the effect of segmentation type on accident modeling results, based on pavement physical characteristics, is also important from another perspective. In similar studies conducted by $[1,3,4]$ on the impact of pavement condition on accident occurrence, fixed-length segments were utilized for the modeling. The studies mentioned above are among the few that specifically examined the safety effects associated with pavement condition, but the effect of segmentation type on the validity of the results was not included in any of the studies. Instead, the focus of their studies was specifically on fixed-length segments.

Chan et al. [2] concentrated on the effects of asphalt pavement conditions on traffic accidents. They developed twenty-one negative binomial (NB) models for different crash types, and these models were calibrated with pavement characteristic variables, including rut depth (RD), international roughness index (IRI), and present serviceability index (PSI).

Two recent articles of certain interests have been published that attempted to simulate accidents based on pavement characteristic variables [3, 4]. First, Jiang et al. [3] examined three types of two-vehicle crashes in terms of pavement management and traffic factors. Subsequently, Jiang et al. [4] correlated pavement management and traffic parameters to accident frequency occurrence. Unlike 
the previous severity models, the new frequency models have a direct relationship with road roughness, that is, less pavement roughness is associated with less accident frequency.

\section{Data description}

Semnan Province has a vital position in Iran's transportation industry. High transportation demand and heavy trucks traffic are reasons for such conditions, although the pavement condition of its rural roads is not appropriate. On the other hand, this Province does not occupy a good position in the ROR accident occurrence ranking. The presence of severe pavement distresses and high ROR/roll-over accidents were the main reasons for selecting this roadway for the development of CPMs.

The correlations between variables were tested, but none of them were eliminated because of the high correlation between them. Variables' statistical significance and goodness-of-fit (GOF) measures were examined in order to consider the models' stability instead of their correlations $[9,11]$.

The details of the statistical characteristics regarding the data on homogeneous and fixed-length segments are shown in Table 1.

\subsection{Accident data}

The single-vehicle ROR accident is applied in cases where an errant vehicle encroaches the roadway. This encroachment may result in three types of outcomes: non-strike incidence, crash with non-fixed objects at the roadside, and collisions involving roadside features and fixed-objects. All these accidents were considered in this study. Between March 20th, 2011 and March 20th, 2012 (that is the year 1390 in Iranian calendar) and among all 373 single- and multi-vehicle crashes, there were 166 single-vehicle ROR accident occurrences in the SemnanTehran direction [18]. Shafabakhsh et al. [19] studied the spatial analysis of frequency and severity of rural accidents on a significant part of the current study and concluded that the frequency of all types of accidents is considerable within $30 \mathrm{~km}$ from Garmsar, but it was apparent that the severity of the accidents, within $10 \mathrm{~km}$ from Semnan, is a more critical situation.

\subsection{Exposure}

In fact, without the exposure parameter, there is indecision in determining the results of CPM because of the impossibility of an accident occurring in the absence of any exposure component(s). Implying that, if the exposure variable(s) is assumed to be equal to zero or not to be recalled, then the expected ROR accident frequency must be equal to zero.

The form of exposure will change based on the type of segmentation. For fixed-length segments, the exposure function contains only AADT as a predictor. On the other hand, Eenink et al. [16] pointed out that the AADT and segment length are explanatory variables in most CPMs for homogeneous segments. One of the frequent exposure units is the million vehicle-kilometer of travel per year. The exposure's function for the $i$ th homogeneous segment is shown in Eq. (1). It is noteworthy at this point that the AADT and length of a given segment were used in exposure as offset variables [17].
Table 1 Details of the study's variables for homogeneous and fixed-length segments

\begin{tabular}{lllllc}
\hline Variables and abbreviations & Units & Min & Mean & Max & Std. Dev \\
\hline Homogeneous segments & & & & & \\
$\quad$ Exposure & & & & & \\
$\quad$ Annual average daily traffic (AADT) & veh/day & 9570 & 9830 & 10,380 & 0.38 \\
$\quad$ Segment length & $\mathrm{km}$ & 0.7 & 2.74 & 6.7 & 1.53 \\
Pavement condition index (PCI) & $\%$ & 12.5 & 69.35 & 69.35 & 30.4 \\
International roughness index (IRI) & $\mathrm{m} / \mathrm{km}$ & 1.39 & 3.67 & 9.47 & 2.29 \\
Riding number (RN) & 1 to 5 & 0.72 & 2.56 & 3.79 & 1.05 \\
Run-off-road (ROR) accidents frequency & No & 0.0 & 1.16 & 6.0 & 1.26 \\
Fixed-length segments & & & & & \\
Exposure: Annual Average Daily Traffic (AADT) & veh/day & 9570 & 9800 & 10,380 & 0.36 \\
Pavement Condition Index (PCI) & $\%$ & 1.26 & 70.9 & 100 & 31.1 \\
International Roughness Index (IRI) & $\mathrm{m} / \mathrm{km}$ & 1.01 & 3.68 & 13.93 & 2.66 \\
Riding Number (RN) & 1 to 5 & 0.18 & 2.61 & 4.08 & 1.12 \\
Run-Off-Road (ROR) Accidents frequency & No & 0.0 & 1.10 & 9.0 & 1.70 \\
\hline
\end{tabular}


Exposure $_{i(\text { Homogenous Segments })}=\frac{A A D T_{i} \times 365 \times \text { Length }_{i}}{10^{6}}$

\subsection{Pavement surface characteristics data}

Pavement condition index $(\mathrm{PCl}) \mathrm{PCl}$ is a numerical indicator, which represents the present condition of a pavement by calculating the observed distresses on its surface. The PCI for the roadway was estimated based on ASTM D6433-11 [20]. The 19 distress types, which were assessed for total length included alligator cracking, bleeding, block cracking, bumps and sags, corrugation, depression, edge cracking, joint reflection, lane/shoulder drop-off, longitudinal and transverse cracking, patching and utility cut patching, polished aggregate, potholes, railroad crossings, rutting, shoving, slippage cracking, swell and also, weathering and raveling.

International roughness index (IRI) The IRI is a mathematical transformation of a longitudinal profile that represents the pavement roughness. This variable relates the movements of a simulated quarter-car to a single longitudinal wheel path profile at a constant speed of $80 \mathrm{~km} / \mathrm{h}$. Measuring method standardized and submitted in ASTM E192608 [21].

Ride number ( $R N)$ The $\mathrm{RN}$ is defined as the ride-ability index of a pavement. It was developed to estimate subjective ride quality from longitudinal profile measurements. The estimation procedure of RN value was certified by ASTM E1489-08 [22].

\section{Methodology}

The methodological issues and processes associated with the establishment, fitting, and evaluation of any crash prediction models (CPMs) are discussed under the following procedure:

- Defining and collecting explanatory variables' data;

- Offering appropriate functional/model forms;

- Running suggested models;

- Evaluating Goodness-Of-Fit (GOF);

- Estimating model errors.

\subsection{Nonlinear negative binomial regression model}

Roque and Cardoso [23] believed that there is no argument to constrain CPMs from following the simple methods (for example, generalized linear models (GLMs)). The crucial issues in analyzing accident events are the accuracy and fitness of the model according to accident data and its variables.

The procedure considered for the development of these models was the forward procedure, which was used to fit ROR accidents into the added variables. Then by considering a proper GOF measure for each model type, the significance of the new model was examined. Several steps were executed and at each stage to estimate the coefficient variables and the dispersion values of the study topic, which became necessary to ascertain whether the suggested functional forms give better GOF values.

The structure of nonlinear CPM, as given in Eq. (2) highlighted that the effects of independent variables were multiplied to the overall accident frequency.

$E(y)=f($ Exposure $) \times g\left(x_{1}\right) \times h\left(x_{2}\right) \times I\left(x_{3}\right)$

where $E(y)$ is the expected single-vehicle ROR accident frequency, and $f, g, h$ and $I$ are the proposed functional forms for each independent variable $\left(x_{i}\right)$.

The Eq. (2) exhibits the multiplicative function of independent variables. There are two types of function relative to an operator. These properties concern how the function is affected by arithmetic operations on its operand. The followings are special examples of a homomorphism on a binary operation:

- Additive function Preserves the addition operation: $f(x$, $y)=f(x)+f(y)$.

- Multiplicative function Preserves the multiplication operation: $f(x, y)=f(x) \times f(y)$.

This study assumed that the multiplicative function gives better GOF results. As the functional form of each independent variable is not defined at the first introduction of the equation, the letter of $f, g, h$ and $/$ represent the function form of each independent variable at each step of forward procedure. The procedure considered for the development of these models was the forward procedure, which was used to fit ROR accidents into the added variables. In fact, letter of $f$, $g, h$ and $/$ are the common names of any functions.

The NLMIXED procedure in SAS was utilized to estimate the coefficients of the functional forms. The ability of NLMIXED to fit nonlinear models necessitated the adoption of this procedure. The NLMIXED procedure maximizes approximation to the log-likelihood integrated over the random effects using some optimizing techniques [24].

The log-likelihood function to be maximized for the negative binomial distribution can be expressed as shown in Eqs. (3) and (4) [25]: 
$L(y, \mu, \kappa)=\sum_{i} I_{i}$

$I_{i}=\log \left(f\left(y_{i}, \mu_{i}, \kappa\right)\right)=y_{i} \cdot \log \left(\kappa \cdot \mu_{i}\right)-\left(y_{i}+1 / \kappa\right) \cdot \log \left(1+\kappa \cdot \mu_{i}\right)+\log \left(\frac{\Gamma\left(y_{i}+1 / \kappa\right)}{\Gamma\left(y_{i}+1\right) \cdot \Gamma(1 / \kappa)}\right)$

previous step. In other words, the LRT value is equal to the GOF value of first model minus the GOF value of second model which have extended the first model by add- where $L$ is the log-likelihood function, $l_{i}$ is the individual contribution to the log-likelihood, $y_{i}$ is the response, $\mu_{i}$ is the estimated mean, and $\kappa$ is the dispersion parameter.

\subsection{Goodness-of-fit (GOF) statistics}

For nonlinear CPMs, two stages are involved in determining the developed models' GOF, namely: (1) core functional form and (2) expanded functional forms. The core functional form represents the relationship between the exposure parameter and single-vehicle ROR accident frequency. The recommended criteria for estimating the models' GOF are scaled deviance $(-2 L L)$, the Akaike information criterion (AIC), a small sample bias-corrected version of $\mathrm{AIC}\left(\mathrm{AIC}_{\mathrm{C}}\right)$, and the Bayesian Information Criterion (BIC) [24].

The AIC has been used to measure the relative GOF. The $\mathrm{AIC}_{C}$ is a finite-sample corrected version of AIC. The $\mathrm{BIC}$ criterion has a similar concept to AIC, which means it introduces a penalty term associated with the number of parameters and the natural logarithm of observations' count. All these measures have a similar rule, implying that the smaller $\mathrm{AIC}, \mathrm{AIC}_{\mathrm{C}}$, and $\mathrm{BIC}$ values indicate a better GOF model [26].

After estimating the likelihood ratio test (LRT) (as defined in Eq. (5)) and the scaled deviance values, the decision on whether the core model is improved by adding new independent variable(s) depend on how much these values were close to the value of $\chi_{0 \cdot 1,\left(d f_{2}-d f_{1}\right)}^{2}$ at the $90 \%$ confidence level, where $d f_{1}$ and $d f_{2}$ are the degrees of freedom (DOFs) for core and modified CPMs, respectively. Several studies have been carried out on the application of LRT, and the scaled deviance measures to test the null hypothesis. The null hypothesis is that all coefficients of the newly added variables are zero when two models are identical, that is to say $H_{0}: \beta_{p+1}=0[10,25]$.

$L R T=-2 \log \left(\frac{\text { Likelihood of core model }}{\text { Likelihood of alternative model }}\right)$

The functions of likelihood are those Goodness-offit (GOF) criteria which include $-2 L L, A I C, A I C C$ and BIC in this study. The definition of LRT considers the difference value between these criteria at any step with the ing new independent variable. In this study, since the BIC criterion penalizes the number of parameters stronger than $\mathrm{AIC}$ and $\mathrm{AIC}_{\mathrm{C}}$ measures, it was chosen to emphasize the practical usefulness of the result.

\subsection{Model error estimates}

To investigate how well the model fits the data, the use of error statistics is practically beneficial. These kinds of GOF measures were used to examine external model validation. The Mean Absolute Error (MAE) criterion in Eq. (6) provides a measure of the average misprediction of the model [17].

$M A E=\frac{1}{n} \sum_{i=1}^{n}\left|O_{i}-E_{i}\right|$

where $n$ is the number of observations, $O_{i}$ is a measure of performance obtained from the field data (that is, observed ROR accidents) and $E_{i}$ is a measure of the performance, which was estimated using the CPMs (that is, estimation of ROR accident using the proposed CPMs). Mainly, Root Mean Squared Error (RMSE) is a common indicator of error and calculates the root of the difference between the estimated and observed values squared, which is given in Eq. (7). Similar to MAE, a result closer to zero represents better data fitness.

$R M S E=\sqrt{\frac{1}{n} \sum_{i=1}^{n}\left(O_{i}-E_{i}\right)^{2}}$

\section{Results}

\subsection{Parameter estimation of homogeneous segments' CPM}

Due to the extensive use of the cumulative differences technique and its functioning as the basis in some studies, the present study made use of the cumulative differences method, as well.

The cumulative differences technique can be used for both continuous and discontinuous variables. The general concept of the cumulative differences technique is shown in Fig. 1. The base value of the segmentation variable for 


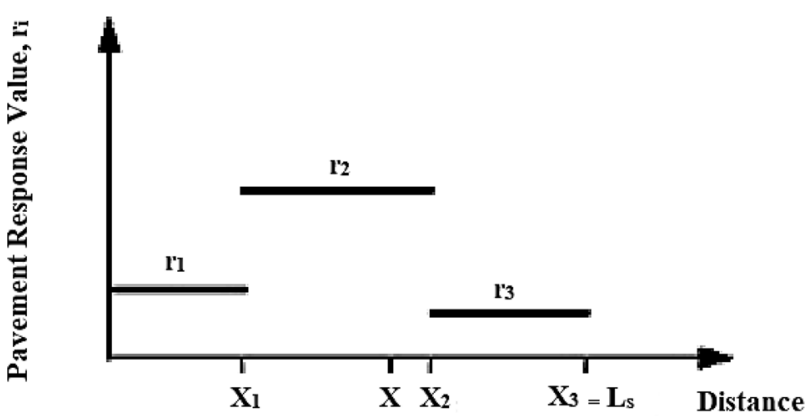

(a)

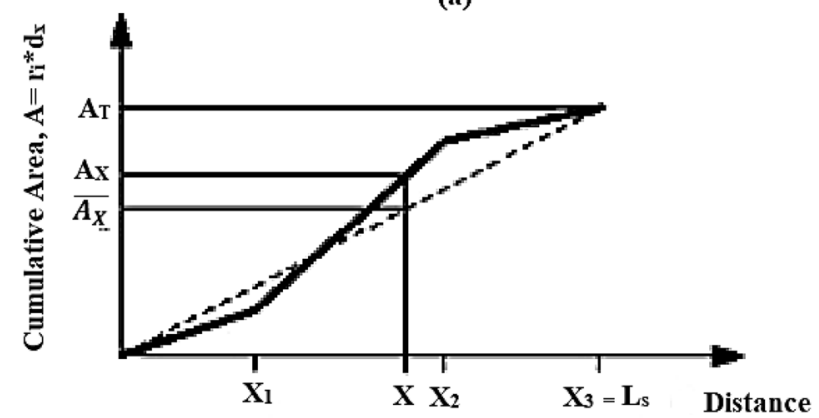

(b)

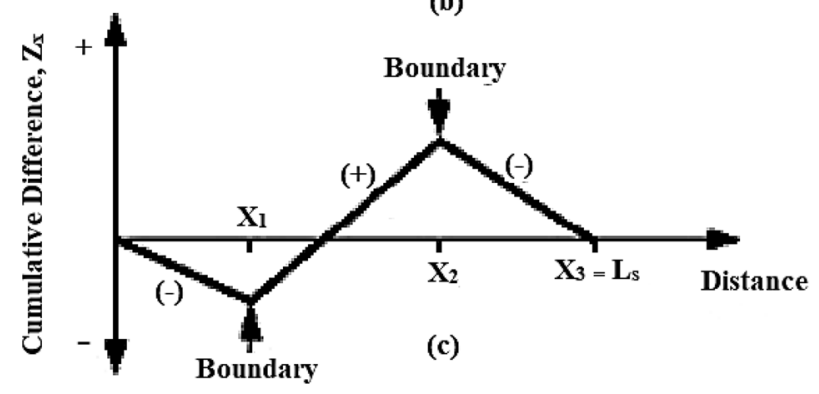

Fig. 1 Details of the study's variables for homogeneous and fixedlength segments [28]

each sample of intervals is shown in Fig. 1a. This variable is assumed to be $\mathrm{PCl}$ in the current study, the value of which is fixed and continuous for all 100-m segments.

In Fig. $1 \mathrm{~b}$, the values of $A_{x}, \bar{r}$ and $\overline{A_{x}}$ were obtained from Eqs. (8), (9) and (10). The slope of cumulative area lines (solid lines) is, in fact, the same value as the variable for the $i^{\text {th }}$ segment, $r_{i}$. The slope of the dotted line equals the overall average value of data [27].
$A_{x}=\int_{0}^{x_{1}} r_{1} \cdot d x+\int_{x_{1}}^{x} r_{2} \cdot d x$

$\bar{r}=\frac{\int_{0}^{x_{1}} r_{1} \cdot d x+\int_{x_{1}}^{x_{2}} r_{2} \cdot d x+\int_{x_{2}}^{x_{3}} r_{3} \cdot d x}{L_{s}}=\frac{A_{T}}{L_{s}}$

$\overline{A_{x}}=\int_{0}^{x} \bar{r} \cdot d x=\bar{r} \cdot x$

Equation (11) can be used to determine the status of changes in the values of the cumulative area. Figure $1 \mathrm{c}$ shows the changing pattern of the cumulative area values, $Z_{x}$, against the distance, $x$. The inflection points of Fig. 1c (where the sign of $Z_{x}$ changes) indicates the range of the domain in which fluctuations of the related variable are homogeneous. In other words, the inflection points determine the range of homogeneous segments [28].

$Z_{x}=A_{x}-\overline{A_{x}}$

The distance between the rural part of Semnan and Tehran roadway is $150 \mathrm{~km}$. This link is a two-lane separated rural highway. The road was separated into 55 individual homogeneous road segments based on the pavement properties, that is, the $\mathrm{PCl}$ component. After determining the homogeneous segments and calculating the values of variables for each segment, the modeling process commenced as follows.

First Step The initial step is developing the core model, which contains only the exposure variables. In using the NLMIXED procedure in SAS, the coefficient of exposure variable converged to the value of 135.39. The achieved was equal to 172.5 and the BIC criterion was 180.5; these values act as references for decision-makers on how newly introduced independent variables could improve the CPM.

Second Step The pavement condition index $(\mathrm{PCl})$, international roughness index (IRI), and ride number (RN) variables were separately added to the core model, and some functional forms were chosen to develop a relationship between run-off-road (ROR) accidents and

Table 2 Estimated coefficients and GOF criteria for independent variables of the second step (Homogeneous Segments)

\begin{tabular}{|c|c|c|c|c|c|c|c|c|c|}
\hline \multirow[t]{2}{*}{ Model name } & \multirow[t]{2}{*}{ Variable } & \multirow[t]{2}{*}{ Best fitted functional form } & \multicolumn{2}{|c|}{ Variables' coefficients } & \multicolumn{4}{|c|}{ Goodness-of-fit criteria } & \multirow[t]{2}{*}{$\kappa(p$ value $)$} \\
\hline & & & $\beta_{1-1}(p$ value $)$ & $\beta_{2-1}(p$ value $)$ & $-2 \mathrm{LL}$ & AIC & AICC & $\mathrm{BIC}$ & \\
\hline $\mathrm{FH}_{2-1}$ & $\mathrm{~g}(\mathrm{PCl})$ & $\mathrm{PCl} . \mathrm{e}^{\beta_{2-1} \cdot \mathrm{PCl}}$ & $8.4118(0.0195)$ & $-0.0193(0.001)$ & 172.0 & 178.0 & 178.5 & 184.0 & $0.5428(0.0397)$ \\
\hline $\mathrm{FH}_{2-2}$ & $g(I R I)$ & $1 /\left(1+\beta_{2-1} \cdot \operatorname{IRI}^{4}\right)$ & $143.06(<.0001)$ & $0.000082(0.5877)$ & 172.0 & 178.0 & 178.5 & 184.1 & $0.5573(0.0353)$ \\
\hline $\mathrm{FH}_{2-3}$ & $g(R N)$ & $R N \cdot e^{\beta_{2-1} \cdot R N}$ & $161.99(0.0330)$ & $-0.3956(0.0197)$ & 172.0 & 178.0 & 178.4 & 184.0 & $0.5546(0.0363)$ \\
\hline
\end{tabular}


independent variables. Among all examined functions, the proposed functional form had the lowest GOF values and as such, has the best fitness to the study data. Thus, the results are shown in Table 2. The $p$-values imply that the $\mathrm{PCl}$ and $\mathrm{RN}$ parameters are significant at the level of $90 \%$, whereas the IRI variable does not satisfy this criterion.

The LRT values for all models were equal to 0.5 and less than 2.71 (that is, the value of $\chi_{0.1,1}^{2}$ ), so these new models did not meet the requirement for significance. However, this little improvement rejects the null hypothesis and is expected to give better GOF by adding other remaining variables. It is critical to mention that in interpreting LRTs, one must exercise care when the value under the null hypothesis falls within the boundary of the variable space [24].

Third Step At this stage, second variables were added to the previous models. Since two models were introduced in the prior stage, this stage should provide two new models. Considering the GOF criteria of the given models in Table 3, it is clear that the Model $\mathrm{FH}_{3-1}$ has the best fit. The model name $\mathrm{FH}_{3-1}$ is the abbreviation of the first suggested functional form for homogeneous segments at the third step. The coefficients' $p$ value emphasizes that the coefficients of Model $\mathrm{FH}_{3-1}$ are significant at about the

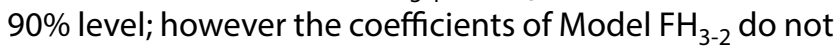
satisfy this criterion.

The LRT value for Model $\mathrm{FH}_{3-1}$ was 2.1 (with regards to Model $\mathrm{FH}_{2-1}$ ), therefore when compared with the $\chi_{0.1,1}^{2}$ value (that is, the value of 2.71 ), the new model significantly improved the prior model.

The final form of the nonlinear CPM for homogeneous segments is defined as shown in Eq. (12).

$$
\begin{aligned}
\text { ROR }- \text { ACC.Homog.Segs. }= & 6.4521 \times \text { Expos. Homog.Segs. } \\
& \times\left(P C l \times e^{-0.03331 . P C l}\right) \times\left(e^{0.4735 . R N}\right)
\end{aligned}
$$

The observed and predicted values of ROR accidents' frequency against the road pavement condition variables are shown in Fig. 2.

\subsection{Parameter estimation of fixed-length segments' CPM}

Regarding the modeling process, this section differs from the previous one due to the development of core models in the first step. Changes in the exposure parameter have been considered as changing factors in the modeling process.

First step In the fixed-length segments' CPM, the exposure parameter includes only the AADT variable; thus, a variety of functional forms need to be evaluated so that a

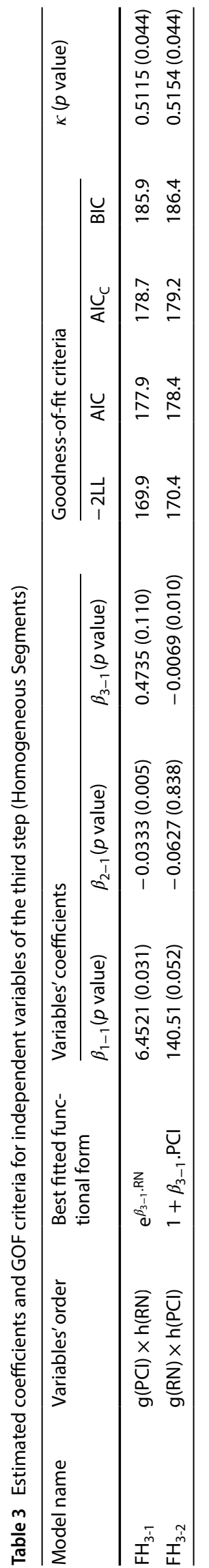

SN Applied Sciences 

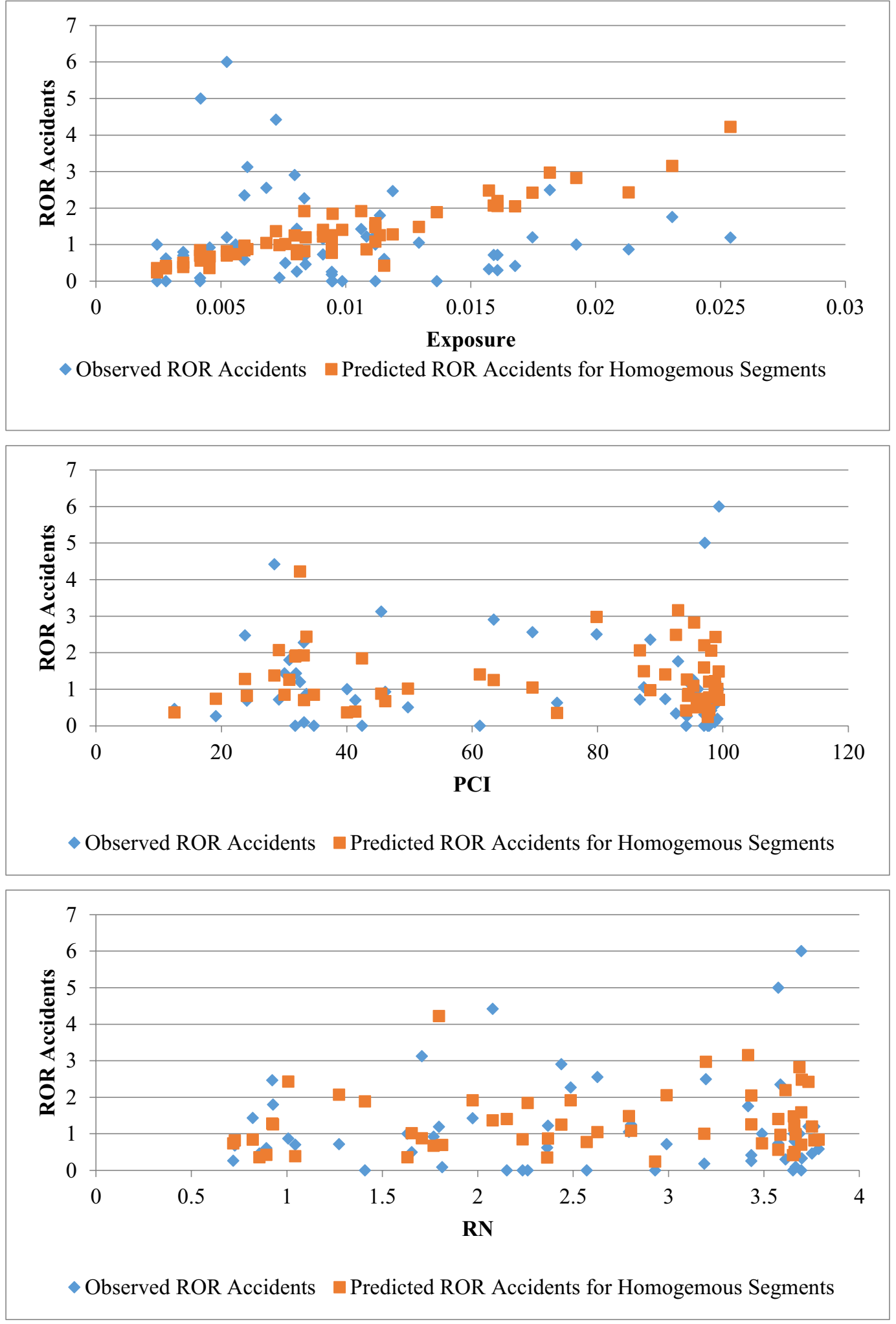

Fig. 2 The scatter of observed and predicted ROR accidents' frequencies in front of pavement variables 
Table 4 Estimated coefficients and GOF criteria for independent variables of the second step (Fixed-length Segments)

\begin{tabular}{|c|c|c|c|c|c|c|c|c|c|}
\hline \multirow[t]{2}{*}{ Model name } & \multirow[t]{2}{*}{ Variable } & \multirow[t]{2}{*}{ Best fitted functional form } & \multicolumn{2}{|c|}{ Variables' coefficients } & \multicolumn{4}{|c|}{ Goodness-of-fit criteria } & \multirow[t]{2}{*}{$\kappa$ ( $p$ value $)$} \\
\hline & & & $\beta_{1-1}(p$ value $)$ & $\beta_{2-1}(p$ value $)$ & $-2 \mathrm{LL}$ & AIC & $\mathrm{AIC}_{\mathrm{C}}$ & $\mathrm{BIC}$ & \\
\hline $\mathrm{FFL}_{2-1}$ & $\mathrm{~g}(\mathrm{PCl})$ & $1+\beta_{2-1} \cdot \mathrm{PCl}$ & $0.1268(<.0001)$ & $-0.0016(0.1105)$ & 4326.7 & 4332.7 & 4332.7 & 4348.7 & $1.7398(<.0001)$ \\
\hline $\mathrm{FFL}_{2-2}$ & $g(I R I)$ & $1+\beta_{2-1} . \mathrm{IRI}$ & $0.1208(<.0001)$ & $-0.01877(0.1605)$ & 4326.8 & 4332.8 & 4332.9 & 4348.8 & $1.7401(<.0001)$ \\
\hline $\mathrm{FFL}_{2-3}$ & $g(R N)$ & $1 /\left(1+\mathrm{e}^{-\beta_{2-1} \cdot \mathrm{RN}}\right)$ & $\begin{array}{l}0.1151 \\
(<.0001)\end{array}$ & $\begin{array}{l}2.7911 \\
(0.0247)\end{array}$ & 4325.1 & 4331.1 & 4331.1 & 4347.1 & $1.7350(<.0001)$ \\
\hline
\end{tabular}

model can be selected, which in addition to being significant, has the best fitness. Based on the use of the NLMIXED process in SAS statistical software, it was found that the exposure function was significant, as shown in Eq. (13), and had the best fit for the ROR accidents' data. The coefficient value of AADT, namely $\beta$, was obtained to be equal to 0.1124 . Values related to $-2 L L$ and $B I C$ criteria were obtained as 4328.4 and 4343 , respectively.

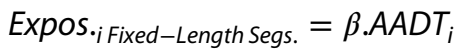

Second step Similar to the second step in the preceding section, the $\mathrm{PCI}, \mathrm{IRI}$, and $\mathrm{RN}$ variables were separately added to the core model and then the changing pattern of GOF and their significance criteria were evaluated. The results of this modeling are shown in Table 4. Here, the $p$-values of the variables show that only $\mathrm{PCl}$ and $\mathrm{RN}$ parameters are significant at the $90 \%$ level. Similar to the modeling of homogeneous segments, the IRI parameter did not fulfill the significance level and therefore, cannot be included in the next step.

In the case of fixed-length segments, by determining LRT values between the core model and the second step models, it can be concluded that the $\mathrm{PCl}$ variable can cause improvement in the fitness of up to 1.7 in the second step in comparison with the core model. This value is approximately 3.3 for the RN parameter, which is higher than the obtained $\chi_{0.1,1}^{2}$ value. However, both $\mathrm{PCl}$ and $\mathrm{RN}$ variables were maintained to develop the models of the third step.

Third step Here, the steps of adding variables to the core model were continued, and the remaining variable was added to the previous model. Investigating the GOF values of the third step's models (Table 5 ) showed that the Model $\mathrm{FFL}_{3-1}$ has the best fit for the accidents' data and the $p$-value relevant to it also confirmed the significance at the $90 \%$ level. The model name $\mathrm{FFL}_{3-1}$ is the abbreviation of the first suggested functional form for fixed-length segments at the third step. The Model $\mathrm{FFL}_{3-1}$ does not only have less fitness but also has some problems regarding the level of significance and as such, cannot be considered as an alternative model.

A comparison of the differences between the BIC values of the Models $\mathrm{FFL}_{2-1}$ and $\mathrm{FFL}_{3-1}$ (or in other words, the LRT value equals 4.9), and the $\chi_{0.1,1}^{2}$ value shows that the addition of the $\mathrm{RN}$ variable to the Model $\mathrm{FFL}_{2-1}$ could cause a significant improvement in the fitness of the new model. The ultimate form of ROR nonlinear CPM for the fixed-length segments was determined as shown in Eq. (14). Figure 3 shows the observed and predicted values of ROR accidents' frequency against the road pavement variables.

$R O R-A C C$.Fixed. Segs. $=0.2582 \times A A D T \times(1-0.00416 \times P C l) \times\left(1 /\left(1+R N^{-0.6794}\right)\right.$ 


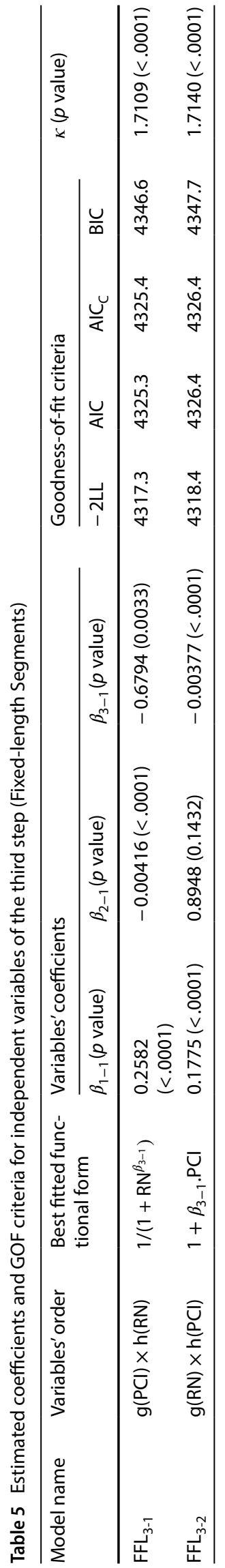

\section{Discussion}

\subsection{Estimation of aggregate goodness-of-fit measurements}

The primary point in the interpretation of Fig. 3 (in comparison with Fig. 2) is that almost all output values of the fixed-length segmentation model fluctuate around 1 , and are not at all consistent with the fluctuations of the observed values of the accidents. However, the comparison of the LRT values for both second and third steps of homogeneous and fixed-length segmentation models indicated that the fixed-length segments model has apparently better position in terms of the degree of improvement. Therefore, it is essential to examine the status of residuals and error in order to attain a more accurate definitive result.

The estimated MAE value for the nonlinear model of homogeneous segments (Eq. (12)) was equal to 1.09, which was increased to about 1.24 for the fixed-length segments (Eq. (14)). Moreover, the RMSE value was computed to be 1.5 in the homogeneous segments model, a value which was increased to about 1.69 for the fixedlength segments model. The comparison of errors shows that, although the model of fixed-length segments had a better fit in some cases, it resulted in an eventual increase in errors.

\subsection{Safety effects of independent variables}

The values of GOF and significance criteria in Eqs. (12) and (14) indicate that the $\mathrm{PCl}$ and $\mathrm{RN}$ variables are statistically significant and fit well to ROR accidents' data (however, Eq. (14), due to having a higher error, is not considered as a suitable option). The method of association between the independent variables and the ROR accidents' frequency can be interpreted by investigating the functional form of the model, as well as the value and sign of coefficients of the independent variables.

In Eq. (12), the positive sign of the coefficient indicated a direct correlation between the explanatory variable and accidents' frequency, while the negative sign of the coefficient indicated the opposite impact of that variable on the frequency of accidents. Since the sign of the $\mathrm{PCl}$ variable coefficient is negative, it can be concluded that the increasing $\mathrm{PCl}$ value (that is, improvement of road pavement condition) can reduce the frequency of ROR accidents. However, the situation is different in the case of an RN variable, which means that the increasing ride quality could lead to a higher frequency of ROR accidents.

Similar results can be obtained with Eq. (12) by investigating the changes of variables on their domains, as 


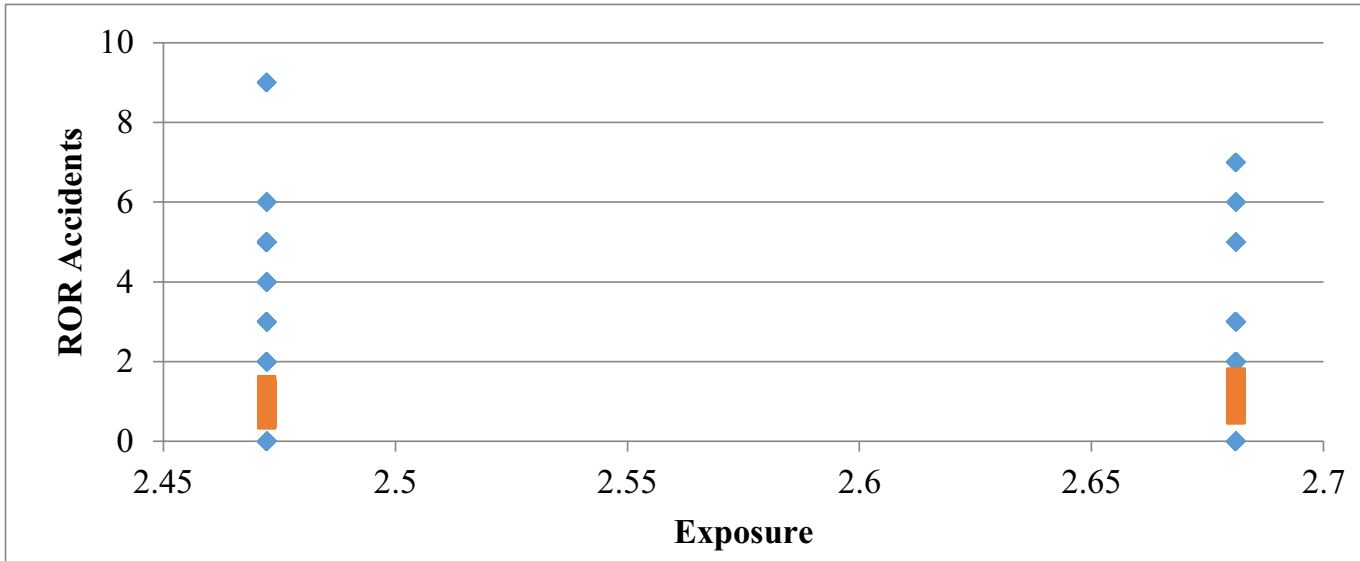

$\diamond$ Observed ROR Accidents $\quad$ Predicted ROR Accidents for Fixed-Length Segments

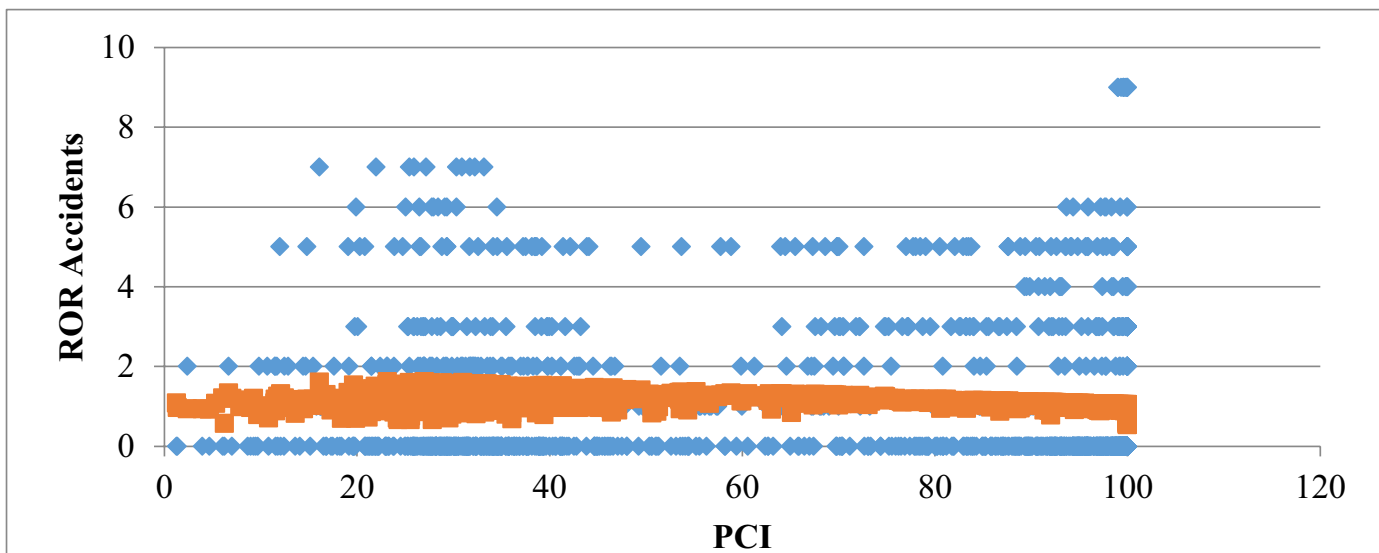

- Observed ROR Accidents $\quad$ Predicted ROR Accidents for Fixed-Length Segments

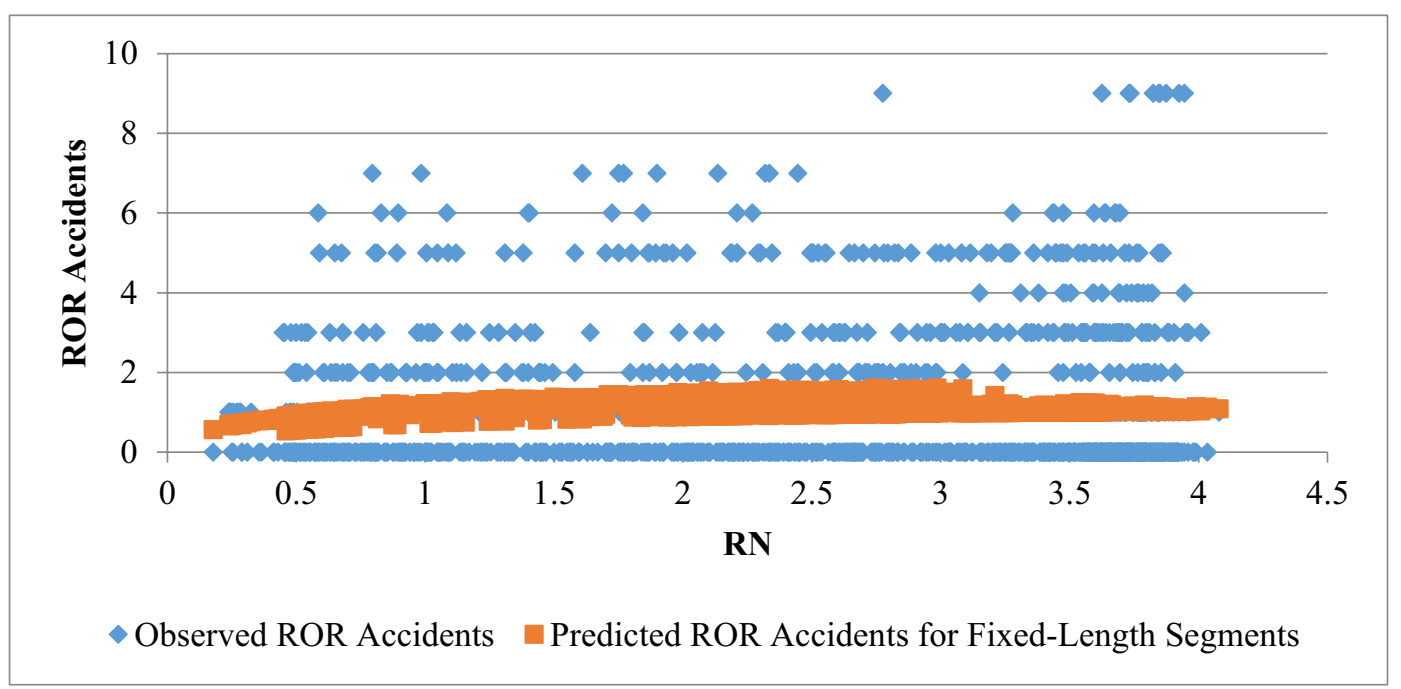

Fig. 3 The scatter of observed and predicted ROR accidents' frequencies in front of pavement variables 
shown in Eq. (14), implying that the $\mathrm{PCl}$ and $\mathrm{RN}$ variables have a direct and adverse impact on the frequency of ROR accidents, respectively.

\section{Conclusions}

Investigating the necessity of segmentation for developing a run-off-road (ROR) crash prediction model (CPM) based on pavement surface characteristics was the aim of this study. Reviewing the recent literature clarified that the fixed-length segmentation was implemented to develop CPMs with pavement variables, whereas the other studies suppose homogeneous segmentation. Concerning CPM literature, it was revealed that homogeneous and fixedlength segmentations are implemented based on assumptions and as such, the importance of segmentation alternatives was not examined particularly.

In this study, the two nonlinear NBR CPMs were developed to investigate the effect of segmentation method on the aggregate goodness of fit measurements of proposed models. In this regard, the homogeneous and fixedlength segmentation procedures were examined. For this purpose, a variety of variables were used which included accident frequency data, AADT and length of segment (as exposure variables), $\mathrm{PCl}$ (as pavement condition variable), $\mathrm{IRI}$ and $\mathrm{RN}$ (as riding quality variables).

Based on the results of the aggregate goodness of fit measurements estimations (include MAE and RMSE measures), it is concluded that the aggregate goodnessof-fit measurements depend meaningfully on the type of segmentation method. The CPM in which had the least errors was the proposed homogeneous segmentation model (that is, Eq. (12)). Therefore, it can be suggested that homogeneous segments should be considered instead of fixed-length segments in developing CPMs, especially for the investigation of specific accident types or particular variable categories. However, both nonlinear ROR accident CPMs were significant and fitted well to ROR accidents' data.

It was also found that regard to the mentioned statistical results for both models, the pavement condition index $(\mathrm{PCl})$ and ride number (RN) variables were statistically significant and associated with the frequency of ROR accidents. It is noteworthy to mention that the sign of the PCI variable coefficient is negative, that means the increasing $\mathrm{PCl}$ value (i.e. improvement of road pavement condition) can reduce the frequency of ROR accidents. Unlike the $\mathrm{PCl}$, the $\mathrm{RN}$ variable had adverse impact which means the increasing ride quality could lead to a higher frequency of ROR accidents.
Of course, this study did not aimed to propose the exact choice of selecting homogeneous or fixed-length segmentation. That means researchers should consider that the CPMs' GOF could be affected by the assumption of selecting fixed-length or homogeneous segments, although results implied that conducting segmentation most likely leads to more reliable outcomes and fits well to data. Regard to several studies which were attempted to develop CPMs by using homogeneous segmentation, the study's findings could be assumed as a general suggestion. As this study was implemented nonlinear NBR model to estimate aggregate goodness of fit measurements, it is recommended to use different methods or statistical models to confirm or examine the effect of segmentation method on the aggregate goodness of fit measurements (external errors).

\section{Compliance with ethical standards}

Conflict of interest The authors declare that there's no conflict of interests that could affect the work reported in this paper.

\section{References}

1. Shankar V, Mannering F, Barfield W (1995) Effects of roadway geometrics and environmental factors on rural freeway accident frequencies. Accid Anal Prev 27(3):371-389. https://doi. org/10.1016/0001-4575(94)00078-Z

2. Chan CY, Huang B, Yan X, Richards $S$ (2010) Investigating effects of asphalt pavement conditions on traffic accidents in Tennessee based on the pavement management system (PMS). J Adv Transp 44(3):150-161. https://doi.org/10.1002/atr.129

3. Jiang X, Huang B, Yan X, Zaretzki RL, Richards S (2013) Two-vehicle injury severity models based on integration of pavement management and traffic engineering factors. Traffic Injury Prev 14(5):544-553. https://doi.org/10.1080/15389588.2012.731547

4. Jiang X, Huang B, Zaretzki RL, Richards S, Yan X (2013) Estimating safety effects of pavement management factors utilizing Bayesian random effect models. Traffic Injury Prev 14(7):766-775. https://doi.org/10.1080/15389588.2012.756582

5. Cenek PD, Davies RB, McLarin MW, Griffith-Jones G, Locke NJ (1997) Road environment and traffic crashes. Research report 79. Transfund, Wellington

6. Costa JO, Maria APJ, Pereira PAA, Freitas EF, Soares FEC (2015) Portuguese two-lane highways: modelling crash frequencies for different temporal and spatial aggregation of crash data. Transport 30:1-12. https://doi.org/10.3846/16484 142.2015.1073619

7. Geyer J, Lankina E, Chan C-Y, Ragland D, Pham T, Sharafsaleh A (2008) Methods for identifying high collision concentration locations for potential safety improvements. Report UCB-ITSPRR-2008-35. University of California, Berkeley

8. Kweon YJ, Kockelman K (2005) Safety effects of speed limit changes: Use of panel models, including speed, use, and design variables. Transp Res Rec JTransp Res Board 1908:148-158. https ://doi.org/10.3141/1908-18 
9. Greibe $P$ (2003) Accident prediction models for urban roads. Accid Anal Prev 35:273-285. https://doi.org/10.1016/S0001 $-4575(02) 00005-2$

10. van Petegema JWH, Wegman F (2014) Analyzing road design risk factors for run-off-road crashes in the Netherlands with crash prediction models. J Saf Res 49:121-127. https://doi. org/10.1016/j.jsr.2014.03.003

11. Cafiso S, D'Agostino C (2013) Investigating the influence of segmentation in estimating safety performance functions for roadway sections. In: 92nd Transportation research board annual meeting, Washington

12. Koorey G (2009) Road data aggregation and sectioning considerations for crash analysis. Transp Res Rec 2103:61-68

13. Turner S, Singh R, Nates G (2012) The next generation of rural road crash prediction models: final report. Research Report 509. NZ Transport Agency, Wellington

14. Akbari M, Shafabakhsh G, Ahadi MR (2020) Single-vehicle runoff-road crash prediction model associated with pavement characteristics. Int J Eng 33(7):1375-1386. https://doi.org/10.5829/ ije.2020.33.07a.25

15. Ambros J, Jurewicz C, Turner S, Kieć M (2018) An international review of challenges and opportunities in development and use of crash prediction models. Eur Transp Res Rev 10(35):1-10. https://doi.org/10.1186/s12544-018-0307-7

16. Eenink R, Reurings $M$, Elvik R, Cardoso J, Wichert S, Stefan $C$ (2008) Accident prediction models and road safety impact assessment: Recommendations for using these tools. Technical report, RIPCORD-ISEREST Deliverable D2. https://ec.europa.eu/ transport/roadsafety_library/publications/ripcord_d02_road_ safety_impact_assessment.pdf

17. Spiegelman CH, Park ES, Rilett LR (2011) Transportation statistics and microsimulation. CRC Press-Taylor and Francis Group, Florida

18. FAVA department (2012) Crashes dataset collection for the year 1390 (Iranian's calendar). Rahvar Police (General Office of Information Technology), Tehran. (In Persian)

19. Shafabakhsh G, Famili, A, Akbari, M (2016) Spatial analysis of data frequency and severity of rural accidents. Transportation Letters Published Online: 08 Mar 2016. https://doi. org/10.1080/19427867.2016.1138605
20. ASTM D6433-11 (2011) Standard practice for roads and parking lots pavement condition index $(\mathrm{PCI})$ surveys. ASTM International, West Conshohocken, PA. https://doi.org/10.1520/D6433

21. ASTM E1926-08 (2015) Standard practice for computing international roughness index (IRI) of roads from longitudinal profile measurements. ASTM International, West Conshohocken, PA. https://doi.org/10.1520/E1926

22. ASTM E1489-08 (2013) Standard practice for computing ride number (RN) of roads from longitudinal profile measurements made by an inertial profile measuring device. ASTM International, West Conshohocken, PA. https://doi.org/10.1520/E1489

23. Roque C, Cardoso JL (2014) Investigating the relationship between run-off-the-road crash frequency and traffic flow through different functional forms. Accid Anal Prev 63:121-132. https://doi.org/10.1016/j.aap.2013.10.034

24. SAS Institute Inc. (2009) SAS/STAT ${ }^{\oplus} 9.2$ user's guide. SAS Institute Inc., Cary, NC

25. Jafari R, Hummer JE (2013) Safety effects of access points near signalized intersections. In: 92nd Annual Meeting of Transportation Research Board. TRB2013, Washington DC. https://trid.trb. org/view/2013/C/1240882

26. Burnham KP, Anderson DR (2010) Model selection and multimodel inference: a practical information-theoretic approach. Springer, New York

27. Cafiso S, Graziano AD (2012) Definition of homogenous sections in road pavement measurements. Proc Soc Behav Sci 53:10691079. https://doi.org/10.1016/j.sbspro.2012.09.956

28. AASHTO (1993) AASHTO guide for design of pavement structures- Appendix J: Analysis unit delineation by cumulative differences. American Association of State Highway and Transportation Official, Washington DC

Publisher's Note Springer Nature remains neutral with regard to jurisdictional claims in published maps and institutional affiliations. 